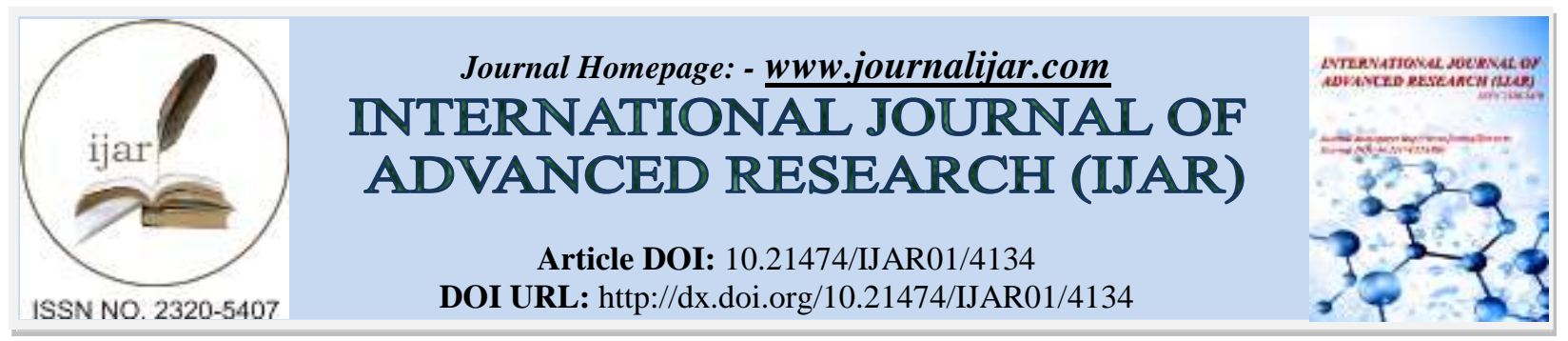

RESEARCH ARTICLE

\title{
ASSESSMENT OF SERUM GONADOTROPINS AND PROLACTIN LEVEL AMONG OLIGO AND AZOOSPERMIA PATIENTS
}

\begin{abstract}
Mohamd Elamin Abdalrazig ${ }^{1}$ and Amar Mohamed Ismail ${ }^{2 *}$
1. Department of Clinical Chemistry, Faculty of Medical Laboratory Sciences, Al-Neelain University, Sudan.

2. Department of Biochemistry and Molecular Biology, Faculty of Sciences and Technology, University of AlNeelain, Sudan.
\end{abstract}

\section{Manuscript Info}

Manuscript History

Final Accepted: 04 April 2017

Published: May 2017

Key words:-

Gonadotropins, prolactin, Oligospermia,

Azoospermia, male infertility, Sudan
Received: 07 March 2017

\begin{abstract}
The aim of current study was to assess serum gonadotropins (LH and FSH) and prolactin in oligo and azoospermia patients in Khartoum State. In a cross-sectional study (n 60) oligospermia, (n 60) azoospermia and (n 60) normospermia, age range from 20 to 51 years old, were included. Sandwich immunochemistry analysis was utilized to measure serum $\mathrm{LH}, \mathrm{FSH}$ and prolactin. BMI was calculated using weight $/ \mathrm{kg}$ divided height $/ \mathrm{m}^{2}$ formula. Of (n 60) oligo and (n 60) azoospermia about $60 \%$ and $63.3 \%$ were found to be overweight. Compared with the normospermia the azoospermia had significantly increased FSH p-value 0.006, while other comparison analyses showed insignificant differences. Furthermore, person's correlation revealed positive association of LH with $\mathrm{FSH} \mathrm{r}=0.661, p$-value $=0.000$, and $\mathrm{LH}$ with prolactin $\mathrm{r}=0.369, p$-value 0.002 , while negative correlation was observed between prolactin and age $\mathrm{r}=0.369, p$-value $=0.004$. In conclusion, two third of oligo and azoospermia are overweight. In azoospermia FSH is higher, thus could magnify impotence and loss of libido related complications in azoospermia patients.
\end{abstract}

Copy Right, IJAR, 2017,. All rights reserved.

\section{Introduction:-}

Oligospermia and azoospermia are the main causes of male infertility or subfertility, which is a reduced ability to achieve a pregnancy while infertility is defined as the complete inability to produce a pregnancy after about one year of unprotected sexual activity $(1,2)$.

Approximately 8 to $15 \%$ of couples are unable to conceive after 1 year of unprotected intercourse. A male factor is solely account of $20 \%$ of infertility, which defined by abnormal semen parameters but may be present even when the semen analysis is normal, semen analysis is the cornerstone of the diagnosis male infertility, since helps to define the severity of the male factors (3-5). Gonadotropins (FSH, LH) and testosterone are the prime regulators of germ cell development. Abnormal spermatogenesis is often associated with altered serum gonadotropins and testosterone, FSH stimulate spermatogenesis whereas LH act on leydig cells in the testes to stimulate the synthesis of testosterone $(6,7)$.

Prolactin excess likely causes hypogonadism by impairing Gonadotropin releasing hormone $(\mathrm{GnRH})$ release and also leads to impotence and loss of libido. Previously researchers investigate the association sperm count, serum

Corresponding Author:- Amar Mohamed Ismail.

Address:- Department of Biochemistry and Molecular Biology, Faculty of Sciences and Technology, Al480 
gonadotropins and testosterone levels oligo and azoospermia in comparison with normospermia, which reported that, significant increased of LH and FSH while decreased testosterone was observed (8-10).

In fact that, male body mass index on sperm parameters concluded that, the total sperm count and sperm motility in overweight and obese men were significantly lower than men with normal BMI (11-13). Furthermore, the objectives of the present study of is to assess gonadotropins and prolactin among oligo and azoospermia patients, this study associate between study parameters and variables (age and BMI), and to explore whether these variables have an association with complication related.

\section{Materials and Methods:-}

In this cross-sectional hospital based study (n 180) men were included, classified as oligospermia (n 60), azoospermia (n 60) age ranged from 20-50 years old and (n 60) normospermia belonging to the same socioeconomic status and age matched as control subjects. These patients and control were randomly selected according to result of the seminal analysis. All included patients diagnosed as infertile for at least two years or more, from Al-Khalifa Suliman specialized hospital in Khartoum state during period from May to December 2015. Patients with diabetes mellitus and/or in hormonal therapy were excluded from this study.

\section{Semen and blood sampling:-}

Semen ejaculate from each subject was obtained by masturbation into a sterile plastic specimen container at the hospital after three days of sexual abstinence. Venous blood samples (about $4 \mathrm{ml}$ ) were collected under aseptic condition. Serum was obtained after centrifugation at $3000 \mathrm{rpm}$ for $5 \mathrm{~min}$ and kept at $-20^{\circ} \mathrm{C}$ until used.

\section{Ethical considerations:-}

This study was approved by the local ethics committee of Al-Neelain University. An informed consent was obtained from each participant in this study.

\section{Sperm count:-}

Brief according to manufacturer semen was analyzed, sperms count $\leq 20$ million $/ \mathrm{mL}$ were considered as oligospermia while those have no sperms in their ejaculates were classified as azoospermia. Absence of sperms was confirmed further by centrifugation of each sample at $10000 \mathrm{rpm}$ for ten min. In proven fertile controls, the sperm count ranged from 21-110 million/ml, this analysis was performed according to WHO criteria (14).

\section{Biochemical measurements:-}

According to the manufacture, two-site immune-enzymometric assay was used, and then hormone present in the test sample (FSH, LH and prolactin) was bound with monoclonal antibody immobilized on amagnetic solid phase and enzyme-labeled monoclonal antibodies in the AIA-PACK. The magnetic beads were washed to remove unbound enzyme-labeled monoclonal antibodies, after incubated with a fluorogenic substrate 4-methylumbelliferyl phosphate (4MUP). The rate of fluorescence produced by the reaction was measured fluorometricly at 365 and $445 \mathrm{~nm}$ using TOSOH Bioscience automated immunoassay analyzer AIA-360.

\section{Statistical analysis:-}

The capture and data analysis were performed using Excel 2010 and SPSS v-21. The variables were described using percentages. Descriptive statistics mean and SD on subject demographics were calculated. $t$-test and ANOVA were employed for comparison, Pearson's correlation was utilized to assess association. Statistical significance was considered at $p$-value $\leq 0.05$.

\section{Results:-}

Demographic data showed, in the sample, $60 \%$ was overweight and $40 \%$ was normal weight out of oligospermia. Moreover, $63.3 \%$ was overweight and $36.7 \%$ in azoospermia, oligospermia and azoospermia most frequency had is overweight, presented in table 1.

Based on ANOVA analysis, we found that, the mean of FSH was significantly higher in azoospermia in comparison with normospermia $p$-value 0.006 , while insignificant difference was observed in oligospermia. Moreover, LH and prolactin of both oligo and azoospermia were revealed insignificant differences when compared with normospermia. 
Person's correlation analyses showed significant positive correlation between (LH and FSH) $\mathrm{r}=0.661 p=0.000$, while significant positive correlation was observed between (LH and Prolactin) $\mathrm{r}=0.389 p=0.002$, moreover correlation between (Prolactin and Age) revealed negative correlation $\mathrm{R}=0.369 p=0.004$.

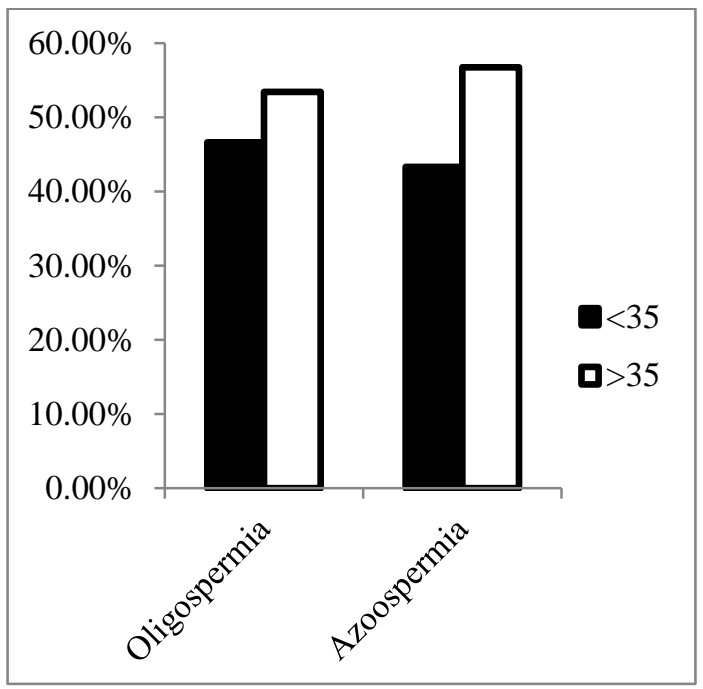

Figure1:- Frequency of sperm count disorders among age groups.

Table 1:- Frequency of overweight subjects in oligo and azoospermia.

\begin{tabular}{|l|c|c|}
\hline Status & BMI & \% \\
\hline Oligospermia & $<26.5$ & $40.0 \%$ \\
& $>26.5$ & $60.0 \%$ \\
\hline Azoospermia & $<26.5$ & $36.7 \%$ \\
& $>26.5$ & $63.3 \%$ \\
\hline
\end{tabular}

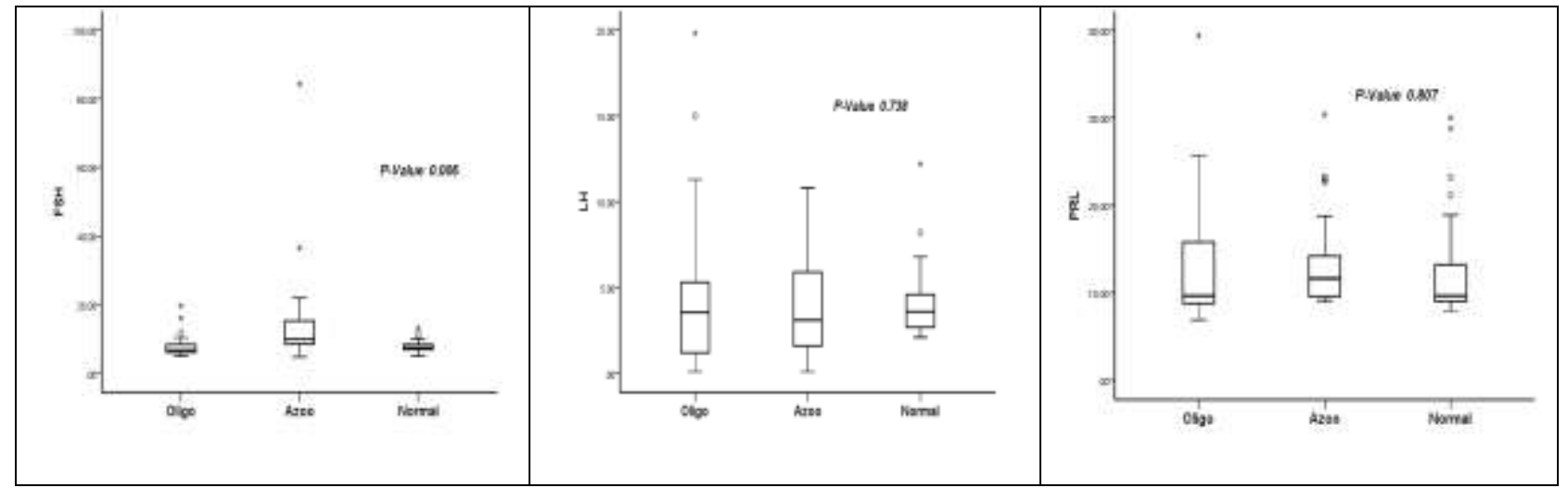

Figure 2:- Shows mean concentration of FSH, LH and prolactin. Results expressed as Mean \pm SD and significant difference considered as $p$-value $\leq 0.05$. 


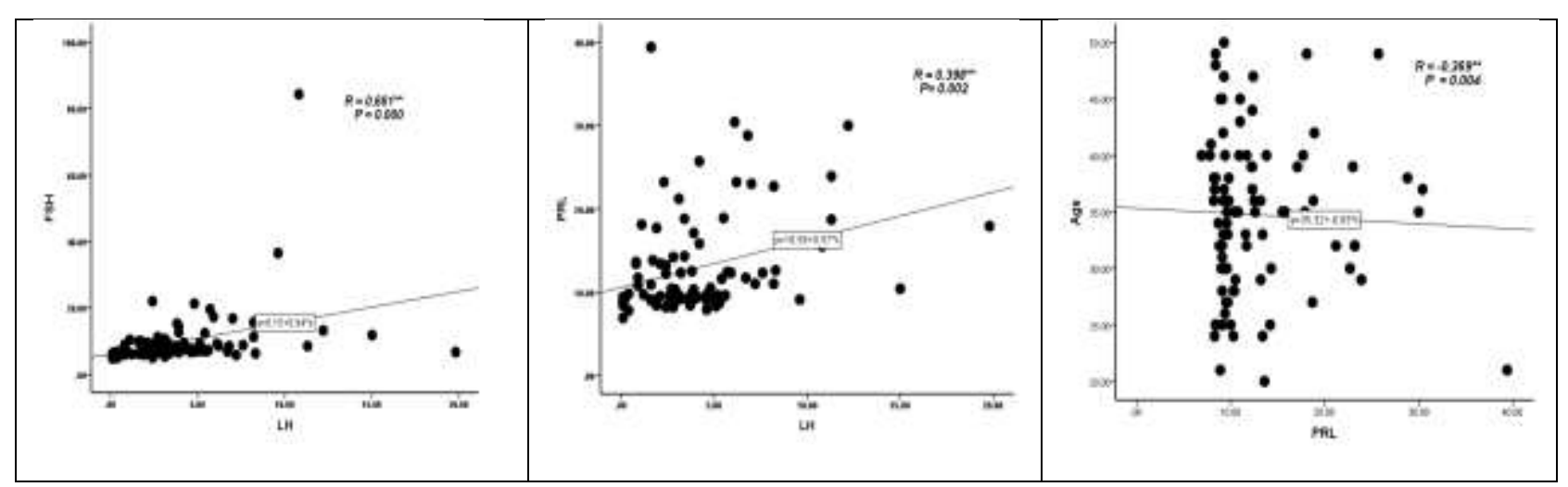

Figure 3:- Person's correlation analyses shows association between (LH and FSH), (LH and Prolactin) and (Prolactin and age), results expressed as $\mathrm{R}=$ regression correlation, $\mathrm{P}=$ strength of correlation.

\section{Discussion:-}

FSH, LH are prime regulators of germ cell development, since production of spermatozoa generally requires the presence of FSH and LH. FSH acts directly on the seminiferous tubules whereas luteinizing hormone stimulates spermatogenesis indirectly via testosterone. Moreover FSH plays a key role in stimulating mitotic and meiotic DNA synthesis in spermatogonia (15). Accordingly the present study aims to assess the level of gonadotropins and prolactin among patients with oligospermia and azoospermia and their correlation with study variables (age and BMI).

The results of this study revealed significant increase in the mean concentration of serum FSH among azoospermic patients in comparison with normospermia with $p$-value 0.019 , while insignificant difference was observed among oligospermia $p$-value 0.998. In fact that, elevated levels of serum FSH with increasing severity of seminiferous epithelial destruction (16), which in teams is a reliable indicator of abnormal spermatogenesis in azoospermia patients. Moreover, it does not eliminate the possibility of obstruction and the capacity for fertility (17). Generally the occurrence of depressed spermatogenesis and elevated FSH levels seems to be a relatively good indicator for the presence of organic disorders of the testis (18).

In addition the result of this study has showed insignificant difference of serum LH concentration in both oligo and azoopermia in comparison with normospermia $p$-value 0.908 and 0.931 respectively, this finding was in agreement with previous report, thus attributed to most oligo and azoospermia disorders associated with germinal epithelial injury with normal testosterone level (19), since it act as feedback inhibitor for LH.

Concurrent with previous findings serum prolactin concentration was insignificantly difference in both oligo and azoospermia in comparison with normospermia $p$-value 0.952 and 0.801 respectively, this finding was in agreement with most previous studies, prolactin is normal in patient with oligo and azoospermia when excluded hyperprolactinemia as causative factor for spermatogenesis and thus infertility (20). Therefore, determination of prolactin in patients with fertility disturbances may be helpful to detect a hyperprolactinemia, which possibly causes inhibition of the spermatogenesis (20).

Pearson's correlation analyses revealed significant positive correlation between FSH and LH (R 0.661, $p$-value 0.000). Interestingly, previous reports showed similar correlations especially with pathological levels (21, 22). Moreover, negative correlation was observed between prolactin level and age of patients (r-0.369, $p$-value 0.004$)$. Evidence from previous finding considered this observation is consistent with altered (increased) dopaminergic tonus in the elderly during the sleep period (23).Positive correlation was observed between prolactin and LH (R 0.398 , $p$-value 0.002). Similarity to ours, Kazanci, showed that, prolactin potentiates the stimulatory effects of LH on testicular steroidogenesis furthermore prolactin activates leydig cells by increasing their responsiveness to LH (24).

\section{Conclusion:-}

In conclusion, two third of oligo and azoospermia are overweight. In azoospermia FSH is higher, thus could magnify impotence and loss of libido related complications in azoospermia patients. Moreover, LH correlates 
positively with FSH and prolactin. The present study support inverse association between prolactin and age in oligo and azoospermia patients. Further case control study is recommended.

\section{Acknowledgements:-}

The author acknowledges sincere thanks to Faculty of medical Laboratory Sciences, Al-Neelain University and AlKhalifa Suliman specialized Hospital staff for the assistance in research work.

\section{Conflict of interest:-}

Authors are declare no conflict of interest

\section{References:-}

1. RajniBala, Vikas Chandra Gupta, Vijay Kumar, A study to evaluate effect of yashadbhasm in oligospermia, world journal of pharmacy and pharmaceutical sciences 2015; 4 (1): 516-520.

2. Jonathan Jarow, Mark Sigman, Peter N. kolettis, Larry R. Lipshultz, R. Dale McClure, Ajay K. Nangia, Cathy Kim Naughton, Gail S. Prins, Jay I. Sandlow, and Peter N. Schlegel. Evaluation of the Azoospermic male: AUA best practice statement, American Urological Association Education and Research 2010; 4-6.

3. Steven Palter, Diagnostic evaluation of the infertile male: committee opinion, American society for reproductive medicine 2015; 103 (3):18-25.

4. Ashok Agarwal, Aditi Mulgund, Alaa Hamada and Michelle Renee Chyatte.A unique view on male infertility around the globe, Reproductive Biology and Endocrinology 2015. 13 (37): 1-9

5. Nazar M. Abdalla, Sudanese male fertility and infections. Sci Parasitol 2011;12 (3):123-129.

6. Tietz fundamentals of clinical chemistry fifth edition, chapter $39,42832,878-881$.

7. Anguilla japonica, Takashi Ohta, Hideyuki Miyake, Chiemi Miura, Hiroyasu Kamei, Katsumi Aida and Takeshi Miura. Follicle-Stimulating Hormone Induces Spermatogenesis Mediated by Androgen Production in Japanese Eel.Biology of Reproduction 2007; 77(6): 970-977.

8. lenau H, Gorewoda I, Niermann H, Relationship between sperm count, serum gonadotropins and testosterone levels in normo-, oligo- and azoospermia. Reproduction 1980; 4 (2):147-156.

9. Lalitha C, SayeeR, Jeyanthi K, Shubha R. Hormonal factors associated with hypogonadismand infertility in males - chromosomal abnormality. IOSR Journal of Dental and Medical Sciences 2013; 10 (1): 71-75

10. Helen L Henderson, Julie Townsend and Domingo J Tortonese. Direct effects of prolactin and dopamine on the gonadotroph response to GnRH. Journal of Endocrinology 2008; 197: 343-350

11. Sekhavat LMoein MR. The effect of male body mass index on sperm parameters, The official journal of the International Society for the Study of the Aging Male; 2010; 13:3 155-158

12. Alshahrani S, Ahmed A-F, Gabr A H, Abalhassan M, Ahmad G. The impact of body mass index on semen parameters in infertile men. Andrologia 2016 doi: 10.1111/and.12549

13. Tang Wen-Hao, Zhuang Xin-Jie, Ma Lu-Lini, QiaoJie, Hong Kai, Zhao Lian-Ming, Liu De-Feng, Mao JiaMing, Zhang Hong-Liang, Zhou Shan-Jie and Jiang Hui. Correlation between body mass index and semen quality in male infertility patients.Turkish Journal of Medical Sciences2015; 45: 1300-1305

14. Cooper T G, Aitken J, Auger J, Baker H W G, Barratt C L R, Behre H.M., Bjorndahl, L., Brazil, C, Jonge, C. D., Doncel, G. F., Franken, D. Haugen, T. B., Hinting, A., Imade, G. E., Kruger, T. F., Odongo, H. O., Noonan, E., Schrader, S. M., Wang, C. C. L., Yeung, W. S. B., Vogelsong, K. M.., Eckardstein, S. V., Mbizvo, M. T., Keizer, M. 2010.WHO Laboratory Manual for the Examination and Processing of Human Semen. WHO Press, Swizerland. 271pp.

15. Ramesh Babu S, Sadhnani M D, Swarna M, Padmavathi P and Reddy P P. Evaluation of FSH, LH and testosterone levels in different subgroups of infertility males Indian Journal of Clinical Biochemistry 2004; 19 (1): 45-49.

16. De Kretser D M. The management of the infertile male. Clin Obstet Gynaecol. 1974; 1(2):409-27.

17. Ahmet Gudeloglu and Sijo J Parekattil. Update in the evaluation of the azoospermic male. Clinics (Sao Paulo); 2013 Feb; 68 (1): 27-34

18. Sina D, Schuhmann R, Abraham R, Taubert HD, Dericks-Tan JS. Increased serum FSH levels correlated with low and high sperm counts in male infertile patients 1975; 7 (1):31-37.

19. McPhaul MJ, Marcelli M, Soppi S: Geneticbasis of endocrine disease: the spectrum of mutations in the androgen receptor gene that cause androgen resistance. Journal of clinical endocrinology metab 1993; 76: 17

20. Der Hautarzt. Prolaction level in the serum of patients with disorders of spermatogenesis. Journal of Dermatology, Venereology, and Related Fields 1978; 29 (2):77-81 
21. Masanori Yamamoto, Satoshi Katsuno, Keisuke Yokoi, HatsukiHiei, and Koji Miyake, Serum inhibin levels in normal men and men with idiopathic infertility. Nagoya J. Med. Sci. 1995; 58: 35-39.

22. Jakiel G, Bakalczuk S, Bokiniec M, Mroczkowski A, Jakubczak A, Swatowski DGinekol Pol. Correlation of pituitary hormones in serum of male patients with fertility problems. Ginekol Pol. 1998; 69(6):413-9.

23. Ferdinand Roelfsema, Hanno Pijl, Daniel M. Keenan, and Johannes D. Veldhuis. Prolactin Secretion in Healthy Adults Is Determined by Gender, Age and Body Mass Index. PLoS One. 2012; 7(2): 1-10.

24. Kazanci G. Prolactin, Gonadotropins, Testosterone and semen parameters. Turkish Journal of Urology $1990 ; 16$ (2): 195-197. 